\title{
Rapid Prototyping of Routing Protocols with Evolving Tuples
}

\author{
Drew Stovall and Christine Julien \\ Mobile and Pervasive Computing Group \\ University of Texas at Austin, Austin TX 78712, USA \\ \{dstovall, c.julien\}@mail.utexas.edu
}

\begin{abstract}
Developing software for dynamic pervasive computing networks can be an intimidating prospect. While much research has focused on developing and describing algorithms and protocols for these environments, the process of deploying these technologies is far from mature or streamlined. Furthermore, the heterogeneity of pervasive computing platforms can make the deployment task unapproachable. In this paper, we describe the evolving tuples model and demonstrate how a simple protocol can be quickly and easily developed. Since the evolving tuples infrastructure serves as a unifying base across heterogeneous platforms, the resulting implementation inherently supports cross-platform deployment, a common scenario for pervasive computing.
\end{abstract}

\section{Introduction}

Since the introduction of ubiquitous computing 14, much research has studied the coordination and collaboration of devices embedded in environments. As predicted, sensing and computing devices are been developed and deployed, and we are continually developing smaller, better, and longer lasting versions. Our rooms, halls, cars, and even parks will eventually be augmented with a plethora of devices to provide and consume all sorts of information.

However, the heterogeneity of devices can impede the creation and maintenance of applications. The variety of platforms to be supported requires an enormous number of protocol and application implementations. This inevitably leads to environments of incompatible, incomplete, and proprietary systems. Additionally, physical access to the devices to update hardware and software leads to massive efforts, making them impractical if not impossible.

Once deployed, this variety of hardware and software is a stumbling block to successful application maintenance. Small alterations to network protocols or node behaviors can cascade into significant code changes. The work required to recompile and redeploy new features can slow development. To address these issues, we introduce the evolving tuples model through which developers can make changes to protocols and applications, run different versions side-by-side, and add features without the cost of traditional redeployment.

In this paper we present the evolving tuples model and examine its use in prototyping behavior for pervasive computing environments. A simple route discovery 
protocol is described in detail to give a practical example of the work, showing the simplicity of prototyping applications using the evolving tuples model.

\section{Background}

Originally introduced as part of the Linda [6] system, a tuple is simply an ordered list of typed data fields. Tuples are collected in a bag-like data-structure called a tuple space. The addition and removal of a tuple from a tuple space is atomic, making it a natural mechanism for buffered communication between parallel processes.

In Linda, a process removing a tuple from the tuple space provides a pattern to which candidate tuples are compared. These patterns take the form of an ordered sequence of actual or formal values. A tuple that matches a pattern has the same number of fields as the pattern, equal values for any actuals, and the same type as any formals.

While forming a simple mechanism for passing data between processes, this design requires that data producers and consumers are maintained together. Any change in tuple format will require a similar change in the patterns used by existing processes. Since a pervasive computing environment typically consists of devices that are not under the control of a single entity, we must assume that tuple formats and tuple patterns will change independently. In our evolving tuples, as in LighTS 1] and ELights [9], fields are tagged with names, enabling us to decouple the tuple and pattern definitions.

Using tuple space systems, data can be effectively communicated between processes administrated by different organizations. However, behavior must still be specified a priori so that an application generating tuples can provide the right data to the consumers. Evolving tuples reduce this level of coupling by directly embedding some of the behavior we expect from nodes into the tuple itself. Specifically, rather then pre-defining the data manipulation recipe to nodes, evolving tuples allow tuple creators to stipulate this behavior at runtime.

\section{The Evolving Tuples Model}

In this section, we describe the evolving tuples model, consisting of three major components: the tuple format, the evolution process, and the standard deployment. A formal specification of many aspects of this model can be found in [12].

\subsection{Evolving Tuple Format}

In addition to the name element described in the previous section, the evolving tuples model adds a formula element to each tuple field. A field's formula specifies how the value is automatically updated or evolved. An evolving tuple is thus a set of tuple fields which comprise a name, a type, a value, and a formula.

A field's formula imparts behavior to the tuple as it passes through the network. Previous to the evolving tuples framework, tuple values were either immutable or altered only according to protocols already deployed to network 
nodes. Though it can be empty or null $(\oslash)$, a field's formula is nominally an arithmetic expression. A few simple logical functions are also provided [12].

These formulas can reference the values of peer fields by name. We also allow expressions to access values of a dictionary-like construct called the evolution context. The evolution context serves as a lookup table for sensor readings, configuration information, and other context related to the tuple's current location. To access values provided by the evolution context, formulas prefix the value's name with "EC." to differentiate them from references to peer fields.

\subsection{Evolution}

When a tuple is evolved, each of its field's formulas are evaluated, and the existing value is replaced with the result. Since formulas combine both the previous value and the values provided in the evolution context, the new value is viewed as an evolution of the field's value. If a field count has a formula of count +1 , the current value of the field would be incremented during an evolution.

\subsection{Standard Deployment Model}

The Evolving Tuples Model includes a reference design that represents the conceptual flow of tuples through each node. While the details of any particular implementation may differ, the externally observable behaviors of each should match those of this reference design. This model contains four components: the receive process and three tuple spaces: inbound, outbound, and application. The model is depicted in Fig. 11

Basic Tuple Exchange. Applications create, initialize, and deposit tuples into the outbound tuple space. Since messages require a destination, the reference model requires, at a minimum, a destination field. The field's value should be initialized to the address of a neighboring node or

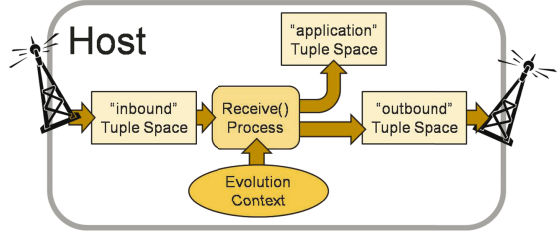

Fig. 1. Flow chart of receive process to the broadcast address. A system process monitors the outbound tuple space, removing tuples and transmitting them to their destinations. If a transmission fails, the tuple is redeposited into the outbound tuple space where it can be selected at a later time for another attempt.

When a host receives a tuple, it is placed in the inbound tuple space. The Receive process removes and evolves each tuple. If, after evolution, the tuple is destined for this node (via the destination field), a copy of the tuple is deposited in the application tuple space. If the tuple needs to be forwarded, the Receive process deposits a copy of the tuple in the outbound tuple space.

Broadcast and Duplicate Elimination. The use of the reserved broadcast address (typically -1) in a tuple's destination field designates that the tuple 
should be sent to every neighboring node. When using this address, the evolving tuples deployment model requires the use of a unique $i d$ field in the tuple to prevent the host from reprocessing tuples it has seen before. Because the tuple $i d$ is simply another field in the tuple, it is possible to alter the value of this id using the field's formula. When a tuple's $i d$ field is changed, it becomes a "new" tuple which will be processed by nodes that have already processed a previous incarnation.

\section{A Routing Protocol}

In this section we demonstrate how route discovery can be performed using the evolving tuples model. In a network of interconnected nodes, a route from one node to another can be found by flooding the network with a "route discovery message". If each node attaches its own address to a list of addresses in the message, a complete hop-by-hop route will be created. When the target node receives the message, a reply message is broadcast across the network to discover a route back to the source. A more complete discussion of route discovery for pervasive networks can be found in 8 .

To build a route discovery tuple, we start with the source and target fields to hold the addresses of the source node and the target node respectively. No formula is specified since these

Table 1. Route Discovery Tuple

\begin{tabular}{|r|c|l|}
\hline Name & Value & Formula \\
\hline source & 0 & $\oslash$ \\
\hline target & 2 & $\oslash$ \\
\hline route & 0 & append $($ route, EC.node) \\
\hline destination & -1 & if ( (source $==$ EC.node, EC.node, -1$)$ \\
\hline id & 0.0 & if (EC.node $==$ target, newUuid ()$, i d)$ \\
\hline
\end{tabular}
are constants throughout the process. The route field carries the accumulated route to which each intermediate node appends its own address (EC.node). The tuple's destination field is used to propagate the tuple to the next node. With one exception, the tuple is always broadcasted and thus the value is usually assigned to the reserved broadcast address -1 . When the tuple is being evolved on the source node, we assign the destination to the source's address (source) to prevent it from further propagation.

Since nodes discard tuples that contain $i d$ 's that have already been processed (to avoid duplicates), we must change the tuple's id when it arrives at the target before it is re-flooded back to the source. We accomplish this using a simple if statement. When the $i d$ formula is evaluated on the target node, the value is replaced with a new id generated by the

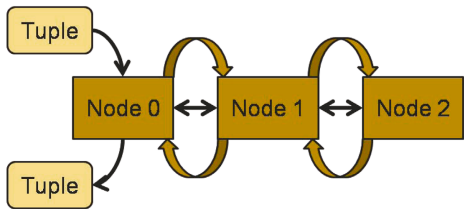

Fig. 2. Example network newUuid() function.

The original tuple deposited by the application is shown in Table 1 (field types have been removed for brevity). In this example, the initiating application resides on a node with address 0 and is attempting to discover a route to a node 
with address 2. If nodes 0, 1, and 2 are interconnected as shown in Fig. 2, the evolution of the tuple's values are shown in Table 2.

When the tuple in Table 1 is deposited into node 0's outbound tuple space, it is broadcasted to neighboring nodes (i.e., Node 1). As the tuple moves through this simple network and is evolved, its fields' values change.

When the tuple is evolved

on node 1 , only the route field is changed, and the tuple is broadcasted again. When the tuple is evolved in node 2 , both the route and id fields are updated. By changing the id field, Table 2. Tuple Values (after evolution on the node heading the column)

\begin{tabular}{|r|c|c|c|c|c|}
\hline Tuple Field & Node 0 & Node 1 & Node 2 & Node 1 & Node 0 \\
\hline id & 0.0 & 0.0 & 2.0 & 2.0 & 2.0 \\
\hline source & 0 & 0 & 0 & 0 & 0 \\
\hline target & 2 & 2 & 2 & 2 & 2 \\
\hline route & 0 & 01 & 012 & 0121 & 01210 \\
\hline
\end{tabular}
we allow the tuple to be received again by node 1 when node 2 broadcasts it again. Node 1 again updates only the route field, and the tuple is passed on to node 0 . Here the destination field is set to 0 to prevent any further flooding of the tuple.

\section{Related Work}

Early tuple space designs [6] and implementations [2] for Linda targeted parallel processing environments. Specifically, the atomic insertion and removal operations on tuple spaces relied on locks provided by shared memory. Lime [11] introduced distributed tuple spaces that provided the same atomicity guarantees across a truly global tuple space spanning many devices in mobile ad hoc networks. This adaptation of tuple spaces allows for an abstract representation of the network underlying a pervasive application but requires that tuples be delivered to consumers without interacting with the "lower levels" of the network. We believe that exposing cross-layer information to tuples in our approach allows for more powerful applications at the cost of a more complex representation.

Mobile agent systems also combine behavior with the data that traverses the network. In an effort to provide a wide range of functionality, undue burden is placed on either the developer or the hosts. Systems like Agilla 5] require the developer to understand very low-level programming languages, while systems like TOTA [10] and MARS 3] require hosts to support high-level languages (i.e., Java). We feel that the evolving tuples model strikes a balance between the skills required to use the system and the capabilities required of the network hosts.

While rooted in different technologies, there are a number of efforts to ease development for pervasive computing applications. Visual programming techniques [13] reduce the learning curve for new developers. Other approaches [7] provide abstraction to manage complexities. Still others address recompilation and redeployment head on through more complex hardware 4]. 


\section{Conclusions and Future Work}

We have presented the evolving tuples model and shown how it can be used to implement a simple route discovery protocol. As we continue to evaluate the model, we anticipate exposing a variety of other domains to which the evolving tuples model is well suited. We feel that our model has the potential to make developing pervasive computing applications more approachable and fruitful.

\section{Acknowledgments}

The authors would like to thank the Center for Excellence in Distributed Global Environments for providing research facilities and the collaborative environment. This research was funded in part by NSF Grant \#CNS-0626777 and AFOSR Grant \#FA9550-07-1-0157. The views and conclusions herein are those of the authors and do not necessarily reflect the views of the sponsoring agencies.

\section{References}

1. Balzarotti, D., Costa, P., Picco, G.P.: The LighTS tuple space framework and its customization for context-aware applications. Int'l Journal on Web Intelligence and Agent Systems (WAIS) 5(2) (2007)

2. Butcher, P.: A behavioural semantics for Linda-2. Software Engineering Journal 6(4), 196-204 (1991)

3. Cabri, G., Leonardi, L., Zambonelli, F.: MARS: A programmable coordination architecture for mobile agents. IEEE Internet Computing 4(4), 26-35 (2000)

4. Dyer, M., Beutel, J., Kalt, T., et al.: Deployment support network - a toolkit for the development of WSNs. In: Langendoen, K.G., Voigt, T. (eds.) EWSN 2007. LNCS, vol. 4373, pp. 195-211. Springer, Heidelberg (2007)

5. Fok, C.-L., Roman, G.-C., Lu, C.: Rapid development and flexible deployment of adaptive wireless sensor network applications. In: Proc. of ICDCS, June 2005, pp. 653-662 (2005)

6. Gelernter, D., Bernstein, A.J.: Distributed communication via global buffer. In: Proc. of PODC, pp. 10-18 (1982)

7. Handorean, R., Payton, J., Julien, C., Roman, G.-C.: Coordination middleware supporting rapid deployment of ad hoc mobile systems. In: Proc. of ICDCS Workshops, May 2003, pp. 362-368 (2003)

8. Johnson, D.B., Maltz, D.A.: Dynamic source routing in ad hoc wireless networks. In: Mobile Computing, vol. 353, Kluwer Academic Publishers, Dordrecht (1996)

9. Julien, C., Roman, G.-C.: EgoSpaces: Facilitating rapid development of contextaware mobile applications. IEEE Trans. on Soft. Eng. 32(5), 281-298 (2006)

10. Mamei, M., Zambonelli, F.: Programming pervasive and mobile computing applications with the TOTA middleware. In: Proc. of PerCom., pp. 263-273 (2004)

11. Murphy, A.L., Picco, G.P., Roman, G.-C.: Lime: A coordination middleware supporting mobility of hosts and agents. ACM TOSEM 15(3), 279-328 (2006)

12. Stovall, D., Julien, C.: Resource discovery with evolving tuples. In: Proc. of ESSPE, September 2007, pp. 1-10 (2007)

13. Weis, T., Knoll, M., Ulbrich, A., Muhl, G., Brandle, A.: Rapid prototyping for pervasive applications. IEEE Pervasive Computing 6(2), 76-84 (2007)

14. Weiser, M.: The computer for the $21^{\text {st }}$ century. Scientific American (September 1991) 